Cita bibliográfica: Morro Fernández, L. (2017). Factores sociofamiliares y estancia hospitalaria: la complejidad de la intervención social en el área de la psiquiatría de agudos. Aplicación de la Escala ECISACH-BCN PSMAR. [Family and social factors and hospital stay: the complexity of social intervention in the area of acute psychiatry. Application of the ECISACH-BCN PSMAR Scale]. Alternativas. Cuadernos de Trabajo Social, 24, 137-160. https://doi.org/10.14198/ ALTERN2017.24.08

\title{
FACTORES SOCIOFAMILIARES Y ESTANCIA HOSPITALARIA: LA COMPLEJIDAD DE LA INTERVENCIÓN SOCIAL EN EL ÁREA DE LA PSIQUIATRIA DE AGUDOS APLICACIÓN DE LA ESCALA ECISACH-BCN PSMAR
}

\author{
FAMILY AND SOCIAL FACTORS AND HOSPITAL STAY: \\ THE COMPLEXITY OF SOCIAL INTERVENTION IN THE AREA OF \\ ACUTE PSYCHIATRY \\ APPLICATION OF THE ECISACH-BCN PSMAR SCALE
} LAURA MORRO FERNÁNDEZ (iD
Hospital del Mar INAD-PSMAR. Passeig Marítim 25-29 Barcelona 08003 91135@parcdesalutmar.cat

\section{Resumen}

Estudiar la complejidad de los determinantes sociales en los procesos de hospitalización psiquiátrica puede ayudar a los profesionales de la salud mental a planificar los recursos que la población requiere durante el ingreso, así como planificar el alta hospitalaria. Hay factores de protección y de riesgo social que hemos de tener en cuenta en el momento de la intervención clínica y social, y que pueden determinar el pronóstico de la enfermedad. Este estudio tiene como objetivo describir los factores sanitarios, sociales y familiares que determinan la complejidad de la intervención social mediante la aplicación de la Escala ECISACH-BCN PSMAR en el área de hospitalización del servicio de psiquiatría del Hospital del Mar de Barcelona, mediante un estudio descriptivo correlacional. Los resultados nos sugieren que la falta de apoyo familiar complica las estancias hospitalarias, teniendo una relación franca la complejidad social y la duración del ingreso hospitalario.

Palabras clave: Complejidad, trabajo social, hospital, planificación del alta, estancia hospitalaria, apoyo familiar.

\begin{abstract}
Studying the complexity of social determinants in psychiatric hospitalization processes can help health professionals plan the resources required by the population during admission, as well as plan for hospital discharge. There are protection and social risk factors that must be considered at the time of clinical and social intervention which can determine disease prognosis. This is a descriptive correlational study that aims to describe the health, social and family factors that determine the complexity of social intervention through applying the ECISACH-BCN PSMAR Scale in psychiatric hospitalization at the Hospital del Mar, Barcelona. The study results suggest that the lack of family support complicates hospital stays and is related to social complexity and length of hospitalization.
\end{abstract}

Keywords: Complexity, social work, hospital, hospital discharge, hospital stay, family support. 


\section{Extended abstract}

\section{Background}

Studying the complexity of social determinants in psychiatric hospitalization processes can help health professionals plan the resources required by the population during hospital admission, as well as plan for discharge.

There are protection and social risk factors that must be considered at the time of clinical and social intervention that can determine disease prognosis.

This is a descriptive correlational study that aims to describe the health, social and family factors that determine the complexity of social intervention through applying the ECISACH-BCN PSMAR Scale in psychiatric hospitalization at the Hospital del Mar, Barcelona.

\section{Introduction}

\section{Health and social determinants}

Throughout history the meaning of the concept of health has changed over time. While the principle of health once referred to the absence of disease, beginning in the middle of the 20th century the concept was expanded upon, and the concept of health has come to refer to a state of complete physical, mental and social welfare.

In 1974, a Canadian report was published that defined the social determinants of health and described how social factors influence the improvement or impairment of population health.

The change in the perception of the concept of health has resulted in changes in the health, economic and social policies of countries, which have begun to take into account the ways in which social determinants affect health and people's lives.

If new models of health also apply to mental health and the treatment of mental health problems, we can say that mental health is something more than the absence of disorder; it is also an integral part of overall health. We thus consider that there is no health without good mental health.

\section{The intervention of medical social work in psychiat-} ric hospitalization

The study of the influence of social factors on hospital care is important for health professionals and administrators in order to measure the factors that affect hospital stays and to properly administer available resources.

Medical social work is a specialty of that has been developed and carried out within the health system (in primary care, social animal care or specialty care) with the objective of facilitating good health, preventing disease and helping the sick and their families to resolve disease-related social and psychosocial problems.

\section{The ECISACH-BCN PSMAR Scale}

Medical Social Work did not have an instrument to measure the complexity of social intervention until in 2012, when a group of medical social workers at the Central Hospital of Lisbon (ordinated by Dr. Serafim) applied the theories of Edgar Morin (regarding the paradigm of complexity) to intervention. They created an instrument that identifies the complexity of an intervention process according to a concrete social situation: the Scale of Social Intervention Complexity in Adults in a Hospital Context (ECISACH).

After the publication of the validation study of ECISACH in Aghatos Magazine, social workers at PSMAR decided to translate and validate the scale in the Spanish context with the name ECISACH-BCN PSMAR. The main objectives of this work follow.

\section{Objectives}

Measure the social intervention complexity in psychiatric hospitalization by investigating factors of mental illness in the family and social context to determine their gravity.

Secondary objectives include:

- Describe the family and social reality of patients admitted in psychiatry.

- Apply the ECISACH-BCN PSMAR scale in the field of psychiatry.

- Explore whether the information provided by the scale is a good measurement of hospital management.

The development of this study was based on two typical assumptions:

- First hypothesis: There is a relationship between the complexity of the intervention and the duration of the hospital stay.

- Second hypothesis: The family serves as a protective factor in relation to the complexity of a social intervention: patients with families present less complexity of social intervention in hospitals, and likewise, patients without families present greater complexity.

\section{Methods}

This is a correlational, descriptive, transversal study, with temporal variables.

The study sample is composed of patients admitted to the acute psychiatry units at Hospital del Mar during the period from January 2017 to May 2017 and that required social intervention.

The data are included in a database made up of one thousand items; it includes sociodemographic data and the twelve items of ECISACH-BCN PSMAR.

Statistical analysis was performed using SPSS. 


\section{Results}

The study results are presented in two areas:

1.- Descriptive analysis of the subscales of the ECISACH-BCN PSMAR Scale: related to the family relationship, $36.1 \%$ of the patients have a good family relationship, the rest presents family distort, being a high percentage, almost $23 \%$, the patients isolated and/or without family. In terms of the analysis of the capacity to mobilize one's own resources and those of the community, the sample presents a high number (68\%) of patients whose families could not offer their own or community resources, so they required professional help.

Related to those patients without a family, 74\% of the patients are without capacity or choose not to mobilize their own or external resources, making the intervention more difficult.

A comparison of the next group (related to the support of the family or its absence) highlights that only $25 \%$ families have the capacity to support the patient, the rest have problems in doing so $(75 \%)$. In the cases of individuals without family, the majority does not have a network of friends or else does not want their help.

In terms of the financial income of the sample, most people have low incomes, and a high number of patients do not have economic income.

Finally, although most patients have adequate housing $(55.4 \%)$, an important percentage (22\%) lives on the street or in poor housing conditions.

The analysis of the data related to the health of the sample reveals a predominance of chronic disease (linked to the diagnosis of psychosis) but that does not affect functionality, since psychiatric patients have a high functionality in daily life activities.

2.- Analysis of the association between the hospital stay and the complexity of the intervention and the association between complexity in patients with family support compared to patients without family support or social ties.

The variable duration of stay was analyzed in relation to possible associated variables, which, according to psychology literature consulted, include age, diagnosis and social complexity. Analysis was carried out using statistical techniques (ANOVA).

Quantitative variables were analyzed first using a Pearson correlation, the duration (days) and the total ECISACH-BCN PSMAR Scale score, more, the age and the duration of the days.

The correlation of days of stay and complexity, was not statistically significant (0.076), however it does suggest a trend.
Thus, we carried out a univariate analysis to know whether there is a relationship between the days of stay and the diagnosis. The F value was high, showing a relationship between the two variables.

We carried out a multiple regression analysis, which showed that intervention complexity is the variable that is statistically significant related to duration of stay, validating the first hypothesis. There is a relationship between the complexity of the intervention and the hospital stay.

To carry out the analysis of the second hypothesis (the family is a protective factor related to the complexity of social intervention), a univariate analysis (Student's t-distribution) was performed between the presence of family support and complexity. The analysis of the two variables shows that the average for patients without families is higher than the average for patients with families and that it is statistically significant, $(\mathrm{m}=39.30 \mathrm{sd} .8 .019 \mathrm{vs} \mathrm{m}=31.87 \mathrm{sd} .9 .131$; $\mathrm{t}=3.4430, \mathrm{p}<0.001)$. Thus, the second hypothesis is also verified.

\section{Conclusions}

The results obtained in this study coincide with studies carried out in other health-related fields, where there is evidence that social relations influences health in adults.

The contributions of this study lie in how these determinants may or may not influence the complexity of medical social work interventions, in terms of the importance of family and social support and the influence on the time of the hospital stay.

\section{Proposals for intervention and new lines of research}

1- Carry out the intervention protocol by medical social workers in people without family support when they require a hospital intervention in psychiatry.

2- Implement the use of the ECISACH-BCN PSMAR Scale widely in hospitalization processes.

Because of the results obtained, a line of research was created to continue to study the relationship between complexity and hospital stay. Its objective is to support the presence of medical social workers in the clinical services that have the highest complexity scores.

A new study with the department of internal medicine will begin soon, and we hope that we can implement the use of the ECISACH-BCN PSMAR Scale as an instrument for use by generalists in the hospital setting. 


\section{Introducción}

En las últimas décadas uno de los objetivos de las políticas sanitarias es determinar la importancia de los factores sociales en la salud y como estos influyen en la gestión y en la planificación de los recursos sanitarios (Lalonde,1981). Ello ha significado un cambio en la concepción del binomio salud-enfermedad, superando las teorías lineales para adoptar modelos basados en la comprensión de las realidades sanitarias de forma compleja, integral, transversal y holística.

El trabajador/a social sanitario/a (a partir de ahora TSS) es pues una pieza clave en las instituciones sanitarias que contemplan la salud de manera integral, ya que es el profesional experto en el estudio y diagnóstico de los factores sociales que afectan a la salud de las personas en los centros sanitarios. La información que proporcionan los/as TSS es fundamental para la gestión y optimización de los recursos de las instituciones sanitarias (Colom, 2012).

Los sistemas de costes intentan dar fórmulas para la gestión de los recursos sanitarios y sociales, pero es necesario alimentar estas bases diagnósticas con los factores que inciden en la complejidad de la realidad de los pacientes y como éstas influyen en su recuperación. Es por lo tanto un reto crear instrumentos que nos ayuden a cuantificar como los factores sociales inciden en la salud de las personas.

En el año 2012, el Departamento de Trabajo Social del Hospital de Lisboa Central creó la Escala ECISACH, traducida y validada a nuestro medio por el equipo de trabajo social del PSMAR (Morro, González, Pineda, Cañete, Casals, Vallvé, Campos, Conti, Moreno, Comas y Prats, 2017). Esta escala posibilita objetivar y cuantificar la afectación de estos factores en la intervención social en el marco de procesos de hospitalización, siendo un instrumento valioso tanto para el trabajador social sanitario como para el gestor hospitalario para la obtención de información sobre su ejercicio profesional y sobre la realidad con la que trabaja.

El objetivo de este estudio es describir los factores sanitarios, sociales y familiares que determinan la complejidad de la intervención social mediante la aplicación de la Escala ECISACH-BCN PSMAR en el área de hospitalización del servicio de psiquiatría del Hospital del Mar de Barcelona.

\section{La salud y su relación con los determinantes sociales}

A lo largo de la historia, el concepto de salud ha ido cambiando de significado, y si bien en un principio era un término antagónico al concepto de enfermedad, desde mediados de siglo XX esta concepción se amplia, entendiendo la salud como un completo bienestar físico, mental y social. 
En 1974, se publica en Canadá el informe Lalonde donde se definen los determinantes de la salud y como los factores sociales influyen en la mejora o en el empeoramiento de la salud de la población (Lamata,1994). Este cambio de percepción significa un cambio también en las políticas sanitarias, económicas y sociales de los países, que empiezan a estudiar cómo afectan los determinantes sociales en la vida y salud de las personas.

La Organización Mundial de la Salud (OMS) define los determinantes sociales de la salud como las circunstancias en que las personas nacen, crecen, viven, trabajan y envejecen, incluido el sistema de salud (OMS, 2007). A partir del modelo explicativo de Lalonde, se construyen diferentes teorías que han tratado de explicar cómo estos determinantes afectan a la salud de las personas (Caballero, Moreno, Sosa, Mitchel, Vega, Columbié, 2012). Diderichsen y la estratificación social (1998), el propio modelo holístico de LaframboiseLalonde (1973), Wilkinson y Marmot (1999) con su aporte desde las políticas públicas, y el más citado, el modelo de influencias en capas de Dahlgren y Whitehead (1991).

Este modelo dispone los determinantes sociales de la salud en diferentes capas superpuestas, representándolos según su proximidad o distancia a los individuos y sus familias. Como muestra la imagen, en el centro sitúa la persona y los factores de carácter individual que se relacionan con su salud (los factores individuales); en la siguiente capa los modos de vida, y en la capa más externa los factores estructurales y políticos (que inciden sobre las inequidades en la salud) y que se relacionan con la economía, las condiciones sociales y las ambientales.

\section{Gráfico 1: MODELO DE DAHLGREN Y WHITEHEAD (1991)}

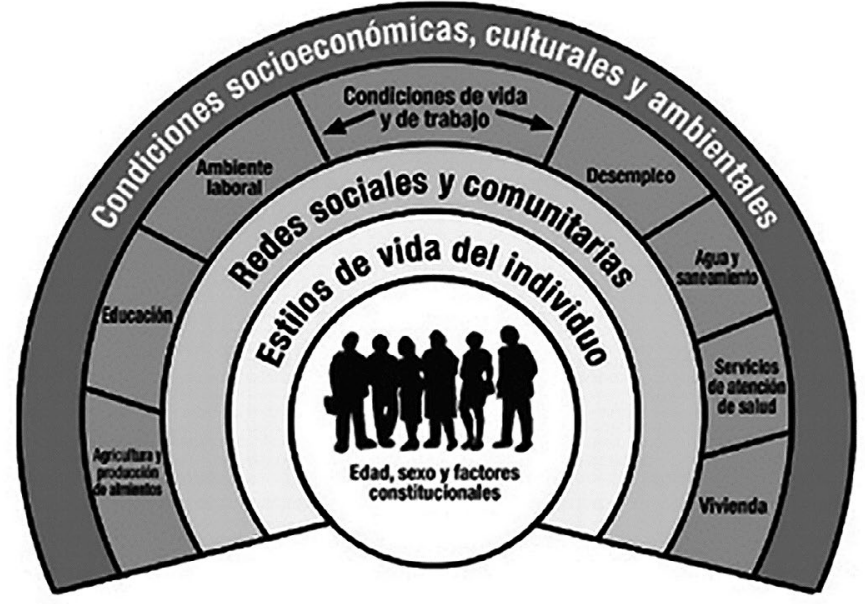

Alternativas. Cuadernos de Trabajo Social, 24, 2017, pp. 137-160 - ISSN 1133-0473 DOI: 10.14198/ALTERN2017.24.08 
En España estas teorías son desarrolladas por la Comisión para reducir las desigualdades en la Salud con el cometido de elaborar una propuesta de medidas de intervención para disminuir estas desigualdades.

\section{Gráfico 2: MARCO CONCEPTUAL DE LOS DETERMINANTES DE LAS DESIGUALDADES SOCIALES EN SALUD. (2010)}

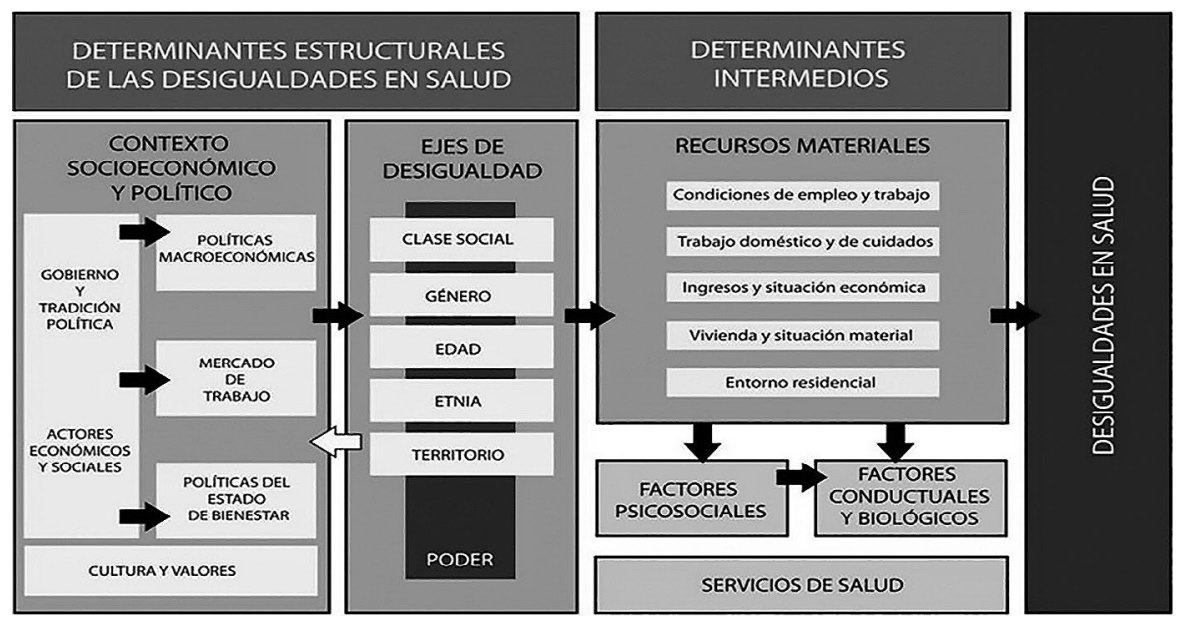

Fuente: Comisión para Reducir las Desigualdades Sociales en Salud en España, 2010

El modelo adoptado por el estado español estructura los factores intervinientes en tres categorías:

1. Contexto socioeconómico y político: la descripción de la distribución de poder y de recursos.

2. Estructura social (incluye los ejes de desigualdad que determinan las jerarquías de poder en la sociedad)

3. Los determinantes intermedios: recursos materiales más los procesos psicosociales y de conducta individual.

Por lo tanto, para establecer políticas de salud, se deben tener en cuenta las posibles patologías de la población (presentes y futuras) así como el impacto de los problemas económicos y sociales, el medio ambiente, las desigualdades, y la cultura de la sociedad.

Todos estos modelos han servido de base para la configuración de un modelo de salud holístico e integral, donde la biología y la genética comparten protagonismo con la situación económica, las redes de apoyo social, el empleo 
y condiciones de trabajo, el entorno psicosocial y demográfico, los estilos de vida, el género y la exclusión social.

\section{Salud mental y determinantes sociales}

Si estos modelos los traspasamos al ámbito de la salud mental y al tratamiento de la misma, podemos decir que la salud mental es algo más que la ausencia de trastorno, es parte integral de la salud, tanto es así que como consideramos que no hay salud sin una buena salud mental (OMS, 2012). Se calcula que una de cada cuatro personas desarrollará un problema de salud mental a lo largo de su vida, siendo más de 400 millones de personas las que experimentan una enfermedad mental en un año. Estas cifras van en aumento, y se prevé que para el año 2020 las enfermedades mentales a nivel mundial serán una de las principales causas de discapacidad.

Esta discapacidad tiene grandes costes sociales y económicos, ya que hay que sumar a los costes sanitarios (tasas muy elevadas de morbilidad y mortalidad), los costes por el desempleo, la falta de productividad, y la cronicidad de quienes la padecen y sus familiares. Si bien no hay una definición única de enfermedad mental, el manual diagnóstico de la Asociación Psiquiátrica Americana (APA), el DSM-5, define los trastornos mentales como los síndromes caracterizados por una alteración clínicamente significativa del estado cognitivo, la regulación emocional o el comportamiento de un individuo, que refleja una disfunción de los procesos psicológicos, biológicos o del desarrollo que subyacen en su función mental (Medrano,2014).

Estos síndromes se caracterizan por alteraciones importantes de la facultad de pensar y de controlar las emociones y la conducta, con una disminución de las capacidades que desarrollan las habilidades sociales y de relación. Los más graves, los llamados trastornos mentales severos (TMS) evolucionaran de forma crónica, con importantes implicaciones personales, pero también familiares y sociales.

Cuando un miembro de una familia tiene dificultades mentales, toda la familia se ve implicada, y debe adaptarse a las nuevas necesidades de su miembro enfermo. Una de las finalidades de las familias es la de cubrir las necesidades básicas de sus miembros, con la finalidad de la socialización de sus miembros en una entidad más amplia, la sociedad donde está establecida (Escartín, 1992).

Diversos estudios relacionan familia y enfermedad crónica, ya que el soporte y el apoyo familiar puede influir en el curso de la enfermedad, tanto de forma negativa como positiva (Ponce, 2007) El afrontamiento que realice la familia delante de la enfermedad de sus miembros determinará en una gran 
parte, el rol que asuma la persona enferma, la conducta de enfermedad. Este término, acuñado por Mechanic y Pilowsky (Galán, Blanco y Pérez, 2000) explica el resultado de la interacción entre variables personales y sociales en el ámbito del enfermar, y aunque es un término en desuso (implicaciones de pasividad del paciente eximido de responsabilidad en sus tratamientos) nos indica la importancia de los determinantes sociales en la salud de las personas.

Es por lo tanto importante en la planificación de estrategias de atención a la salud mental contar con líneas de apoyo y soporte a los enfermos, pero también a las familias y al soporte que la sociedad deberá brindarles. Las políticas sanitarias de atención a la salud mental deben tener en cuenta, por lo tanto, los factores que van a influir en la previsión de las enfermedades mentales, factores biológicos, pero también sociales, laborales, económicos y culturales. Estas políticas de atención a la salud mental de la población han evolucionado en el último siglo, de la mano de la investigación y de la aplicación de nuevas metodologías de investigación en las neurociencias.

La psiquiatría es una de las especialidades médicas que más ha tenido en cuenta la influencia de los factores familiares y sociales, y como estos influyen positiva o negativamente en el individuo, existiendo una voluntad de integrar los distintos elementos biopsicosociales que condicionan la patología psíquica (Vallejo,2002).

La consideración de estos factores por parte de la psiquiatría también ha condicionado los profesionales que conforman los equipos que tratan las enfermedades mentales. Estos son equipos multidisciplinares, donde en la mayoría de los casos nos encontramos con la presencia de profesionales del trabajo social, pudiendo decir que es una de las primeras especialidades médicas en incorporar a TSS en sus equipos.

\section{Trabajo social sanitario en el ámbito de la salud mental}

El TSS es la especialidad del Trabajo Social que se desarrolla y se ejerce dentro del sistema sanitario (atención primaria, la atención socio sanitaria o la atención especializada) y cuyo objetivo es de facilitar una buena salud, prevenir la enfermedad y ayudar a los enfermos y a sus familias a resolver los problemas sociales y psicosociales relacionados con la enfermedad (Barker,1991).

Si recordamos los inicios del trabajo social sanitario, con los esfuerzos del Dr. Cabot (Massachussets 1903-1905) por incorporar a las trabajadoras sociales en sus equipos, también debemos recordar que al unísono, el Dr. Sotuer y el Dr. Meyer pocos años después (1904-1913) hacían lo mismo en las instituciones psiquiátricas de la misma ciudad, junto a Mary C. Garret, una de las primeras trabajadoras sociales del área psiquiátrica, tratando de dar una respuesta 
socializadora a la cronicidad de los trastornos mentales y a la masificación de los manicomios (Garcés, 2010). Y si bien, en un principio el trabajo social tenía un marcado acento católico-asistencialista, en los años 70 evoluciona como disciplina de la mano de las nuevas corrientes de la psiquiatría (movimiento de reforma inspirados en los modelos italianos de la anti psiquiatría de Basaglia), transformándose en agente de cambio y generador de derechos de los enfermos mentales.

Actualmente los TSS forman parte de los equipos de atención de salud mental, y podemos vertebrar sus funciones en varios ejes (atención directa, e indirecta, coordinación, gestión del caso, etc.), pero con un objetivo principal: la elaboración del diagnóstico social sanitario, con el que planificará su intervención y la del equipo. También se ejercen funciones de planificación y promoción de la salud del individuo y su comunidad, así como acciones de formación docencia e investigación. Y si bien, la Ley 44/2003, de 21 de noviembre de Ordenación de las Profesiones Sanitarias, no incluye entre los profesionales sanitarios al trabajador social, en la práctica, los TSS se encuentran presentes en los dispositivos de salud mental, desde la atención primaria hasta la hospitalización, pasando por los servicios de rehabilitación, con el objetivo de impulsar, fortalecer y rescatar los aspectos más sanos de los pacientes y de sus familias, mediante el trabajo social clínico (Ituarte, 2017), aportando el diagnostico social sanitario al plan de intervención integral que realizan todos los miembros del equipo multidisciplinar (Colom, 2012).

\section{Diagnóstico social sanitario: la intervención del TSS desde la perspectiva de las teorías de la complejidad}

El entorno en el que debe intervenir el TSS en el área de Psiquiatría es complejo y diverso, donde se interrelacionan los determinantes de la salud, los factores personales, familiares y sociales de la persona y la enfermedad mental. Por ello es necesario aplicar un marco teórico que contemple esta transversalidad y complejidad de los hechos, que acepte la coexistencia de las diferentes posiciones de la persona enferma, de su familia y su entorno, para poder realizar un diagnóstico social sanitario ajustado a su realidad.

Edgar Morin, sociólogo francés, nos ofrece esta posibilidad de mirar de otra forma las realidades sociales mediante el Paradigma de la Complejidad, modelo teórico presentado en su obra El Método. En esta obra Morin expone que no hay realidades excluyentes, sino que la realidad se compone de sistemas totales complementarios y contradictorios, que no pueden abarcarse ni comprenderse si no es asumiendo su complejidad intrínseca, donde el individuo no se entiende sino es en relación con otros y con su entorno. 
Este autor define (los principios básicos del pensamiento complejo y que podemos aplicar a la comprensión de la realidad sanitaria (Morin, 1994):

1. El principio sistémico u organizacional: donde se relaciona las partes con el todo.

2. El principio Holo gramático: las partes están en el todo, y el todo en las partes

3. El principio reactivo: la causa actúa sobre el efecto y el efecto sobre la causa

4. El principio recursivo: la auto organización y autoproducción.

5. El principio de autonomía y dependencia del medio

6. El principio dialógico, que integra lo antagónico como complementario

7. Y por último el de reintroducción del sujeto como creador del pensamiento.

Esta visión antroposocial, donde se incluye las dimensiones de las ciencias naturales y las ciencias sociales supone una construcción teórica muy rica para el TSS, que se nutre de ambas ciencias para construir su conocimiento (Cordero y Blanco, 2004). Podríamos decir que está en el ADN de los TSS esta visión de la complejidad que nos propone Morin, ya que los TSS son los profesionales del equipo que aportan mediante el diagnostico social sanitario esta visión compleja de la realidad de la persona enferma.

El diagnostico social sanitario contempla a la persona íntegramente, desde su «yo» (personalidad) pasando por su entorno más cercano (familia), su entorno (medio, contexto donde vive) y la enfermedad, considerando ésta como un factor disruptivo que va a cambiar todas las dinámicas internas y externas (su psique, sus relaciones familiares, laborales y sociales) y que determinarán su futuro (Colom, 2012). Para realizar el estudio diagnostico desde la disciplina del TSS se utilizan diferentes herramientas, entre las más relevantes: la entrevista clínica (ya sea al paciente y/o a su familia o personas de su entorno), la observación, las encuestas, y las escalas.

Las escalas son instrumentos científicos que sirven para medir y representar el estado de una o unas variables en un momento determinado. El Trabajo Social tiene diferentes escalas que miden diferentes variables, como por ejemplo el riesgo social, la exclusión social, la funcionalidad, la claudicación familiar, el apoyo social, etc. y que nos sirven de base para por un lado conocer situaciones concretas, y por otro poder compararlas de una manera objetiva con otras situaciones para llevar a cabo una teorización deductiva generando un conocimiento abstracto basado en la práctica clínica. 


\section{La escala ECISACH-BCN PSMAR}

En el ámbito del TSS no se disponía de un instrumento que midiera la complejidad de la intervención social hasta que en 2012, un grupo de trabajadoras sociales sanitarias del Hospital Central de Lisboa (Sefarim,2012) aplicaron las teorías descritas por Edgar Morin (el paradigma de la complejidad) a la intervención desde el TSS, creando un instrumento que identifica la complejidad de un proceso de intervención según una situación social concreta, la Escala de Complejidad de la Intervención Social con Adultos en un Contexto Hospitalario, en adelante Escala ECISACH. Esta escala portuguesa es utilizada en los centros hospitalarios de Portugal, y es un instrumento de calidad de las diferentes instituciones que lo aplican. Se ha traducido y validado esta escala en el contexto español con el nombre de ECISACH-BCN PSMAR (Morro, González, Pineda, Cañete, Casals, Vallvé, Campos, Conti, Moreno, Comas y Prats, 2017)

\section{Objetivo del estudio}

Describir los factores que determinan la complejidad de la intervención social de los pacientes ingresados en una unidad de psiquiatría hospitalaria mediante la aplicación de la Escala ECISACH-BCN PSMAR.

\section{Objetivo principal}

Medir la complejidad de la intervención social en la hospitalización psiquiátrica investigando que factores de la enfermedad mental y de su contexto familiar y social determinan su gravedad.

\section{Objetivos secundarios}

- Describir la realidad familiar y social de los pacientes ingresados en Psiquiatría.

- Aplicar la Escala ECISACH-BCN PSMAR en el ámbito de la psiquiatría

- Explorar si la información que proporciona la Escala es un buen instrumento para la gestión hospitalaria.

\section{Hipótesis}

Las hipótesis de tipo correlacional que se han elaborado durante el estudio son:

I. Existe una relación entre la complejidad de la intervención y la duración de la estancia hospitalaria 
II. La familia es un factor protector frente a la complejidad de la intervención social: los pacientes con familia presentan menor complejidad de la intervención social en los ingresos hospitalarios y al revés, los pacientes sin familia presentan mayor complejidad.

\section{Metodología: Diseño del estudio}

Se trata de un estudio descriptivo correlacional, de carácter transversal, que estudia las variables en un espacio de tiempo corto (el ingreso en la unidad psiquiátrica de agudos), y que tiene en cuenta la relación entre los resultados de la escala ECISAH-BCN PSMAR con las variables socio-demográficas y clínicas de la muestra.

Los estudios correlacionales determinan si dos variables están correlacionadas o no, analizando si un aumento o disminución en una variable coincide con un aumento o disminución en la otra variable (Hernández, 2007). Primero se miden las variables y luego, mediante la aplicación de técnicas estadísticas, se estima si hay correlación o no. Si bien es un tipo de estudio que no establece de forma directa relaciones causales, aporta posibles causas de las mismas.

Hay diferentes tipos de correlaciones: las positivas, las negativas y la no correlación. Esta investigación se ha centrado en las positivas, que pueden aportar una mayor información a las hipótesis.

El estudio se ha realizado en el servicio de Psiquiatría del Hospital del Mar de Barcelona. Este servicio de agudos consta de dos unidades hospitalarias: la unidad 07 (con 7 camas) y la unidad 80 (35 camas).

La población que constituye la muestra del estudio está formada por aquellos pacientes que ingresaron en las unidades de agudos de Psiquiatría del Hospital del Mar durante el periodo comprendido de enero a mayo de 2017 y que han requerido de intervención social por parte del TSS, con una participación final de 83 personas. ( $\mathrm{N}=83$ )

Los datos de la muestra se recogen durante el proceso de intervención social que realiza el TSS durante el tiempo de ingreso del paciente. Tras el alta del paciente de la unidad de Psiquiatría se dispone de la puntuación total de la escala ECISACH-BCN PSMAR, que se completa al finalizar el ingreso hospitalario.

Esta escala consta de 12 ítems agrupados en tres subescalas o variables:

- la primera es el marco sociofamiliar del paciente analizándose el vínculo familiar, la capacidad de movilización de recursos, la disponibilidad de apoyo, los ingresos y la vivienda.

- la segunda variable la situación clínica, que analiza el estado clínico del paciente y su autonomía. 
- la tercera, analiza el proceso de intervención social, contabilizando el número de atenciones, de contactos o coordinaciones, la disponibilidad de apoyo y el tiempo de resolución del caso.

Cada ítem se evalúa con una escala numérica del 1 al 5 siendo el 1 la situación ideal y el 5 la situación más compleja.

La puntuación total se obtiene de la suma de las puntuaciones de las subescalas con unas puntuaciones repartidas en cinco tramos que describen de menor a mayor la complejidad de la intervención: sin complejidad, complejidad baja, complejidad intermedia, alta complejidad y complejidad muy alta.

Todos estos datos se compilan en una base de datos que consta de ítems de carácter sociodemográficos (edad, género, fecha de ingreso y de alta), datos clínicos (diagnóstico psiquiátrico expresado en CIE-9) y los doce ítems relacionados con ECISACH-BCN PSMAR.

Se ha realizado un análisis estadístico mediante el programa estadístico SPSS (versión 24.0), tanto descriptivo como analítico univariante (correlación de Pearson, t-student y ANOVA y multivariante (regresión múltiple) para testar las hipótesis a estudio.

\section{Resultados}

Durante el periodo de estudio, de enero a mayo del 2017, se ha intervenido a nivel social en 109 casos. Esto significa un $43.4 \%$ del total de ingresos de psiquiatría (con una N. total de 251).

De los 109 casos, sólo se ha podido incluir en el estudio 83, ya que los 26 restantes se encontraban aun en procesos activos de hospitalización en el momento del análisis estadístico. Esto significó la imposibilidad de obtener las variables correspondientes a la Escala ECISACH-BCN PSMAR (que se suman al alta hospitalaria) así como los ítems de destino al alta y duración del ingreso, por lo que estos casos han tenido que ser descartados.

La presentación de los resultados se vertebra en dos ejes:

1. Análisis descriptivo de las variables cualitativas y cuantitativas sociodemográficas clínicas y de puntuación total de la escala ECISACH-BCN PSMAR.

2. Análisis descriptivo de las subescalas de la Escala ECISACH-BCN PSMAR.

3. Comparación entre estancia hospitalaria, complejidad social y apoyo familiar. 


\section{Descripción de las variables cualitativas y cuantitativas}

Para el análisis cuantitativo de los datos se han utilizado índices de estadística descriptiva como la media, la desviación estándar para las variables cuantitativas (edad, días de ingreso y la puntuación total de la Escala ECISACH) y los porcentajes en las variables cualitativas: sexo, diagnostico, nacionalidad, derivación al alta.

En la tabla 1 se muestra la media y desviación estándar de las variables cuantitativas: la edad, los días de ingreso y la puntuación total de la Escalad ECISACH-BCN PSMAR.

En cuanto a la edad, la cifra apunta a edad bastante inferior a las muestras de hospitalización general (diferentes estudios, como el Estudio Epine, sitúan la edad media en hospitalización por encima de los sesenta años)

También cabe destacar que la duración de los ingresos en el servicio es extensa, con 24 días de media (la estancia Gold Estándar en Psiquiatría es de 16 días).

La media de la puntuación total de complejidad en la intervención social mediante la escala ECISACH se ha situado en el rango de la complejidad intermedia, y tratándose de casos donde se ha solicitado la intervención de la TSS no es muy alto.

Tabla 1: DESCRIPCIÓN DE VARIABLES CUANTITATIVAS

\begin{tabular}{|l|l|l|l|}
\hline & N & Media & Desviación Estándar \\
\hline Edad & 83 & $\mathbf{4 8 , 2 0}$ & 19,366 \\
\hline Días ingreso & 83 & $\mathbf{2 4 , 6 0}$ & 13,956 \\
\hline $\begin{array}{l}\text { Puntuación total ECISACH_BCN } \\
\text { PSMAR }\end{array}$ & 83 & $\mathbf{3 3 , 5 2}$ & 10,055 \\
\hline N Valida & 83 & & \\
\hline
\end{tabular}

Respecto a las variables cualitativas, hay un predominio muy ligero de hombres (53\%), hecho común en las unidades de atención psiquiátrica. En referencia a los diagnósticos, ante la diversidad de los diagnósticos hallados en la muestra (codificados en CIE9), se ha realizado una reducción diagnóstica, agrupando los trastornos psicóticos y bipolares con el modelo de psicosis única (Peralta, Basterra, Zandio y Cuesta,2008).

Los resultados nos indican un claro predominio de los trastornos psicóticos en los ingresos en la unidad de agudos ( $88 \%$ de los ingresados). En cuanto a las 
derivaciones al alta, hay un alto porcentaje de pacientes (67\%) que requieren de prolongar su estancia hospitalaria en un recurso de media estancia (subagudos) por la necesidad de estabilización clínica, frente al $43 \%$ de pacientes que son dados de alta al domicilio.

\section{Análisis descriptivo de las subescalas de la escala ECISACH-BCN PSMAR}

Como ya se ha comentado anteriormente, la Escala ECISACH-BCN PSMAR consta de 12 ítems agrupados en tres subescalas: marco sociofamiliar, salud e intervención social. A continuación, se presenta el análisis descriptivo de estos ítems, que proporcionan una información muy valiosa de la muestra (tanto a nivel social como de gestión de recursos).

Marco socio-familiar: En cuanto a las relaciones familiares, si bien un $36.1 \%$ de los pacientes mantiene una buena relación familiar, el $41 \%$ de los pacientes presenta distocia familiar, siendo un alto porcentaje, casi un $23 \%$, los pacientes aislados y/o sin familia. Al analizar la capacidad de estas familias para movilizar sus recursos y los de la comunidad, la muestra presenta un número alto (68\%) de pacientes cuyas familias no pueden ofrecerles recursos propios o de la comunidad, por lo que requieren de ayuda profesional.

Si nos fijamos en el grupo de pacientes sin familia, los porcentajes empeoran, encontrándonos con un $74 \%$ de los pacientes sin capacidad o con rechazo para la movilización de recursos propios o externos, lo que dificultará la intervención.

Al comparar el siguiente grupo (relacionado con el apoyo de la familia o su ausencia), resalta que sólo en un $25 \%$ de los casos las familias tienen capacidad para apoyar al paciente, presentando problemas el resto (75\%).

En los casos de individuos sin familia, la mayoría no tienen red de amigos o rechazan su ayuda (60.9\%).

Cuando se examinan los datos de ingresos económicos de la muestra, se constata que se trata de personas con rentas bajas, y con un alto número de pacientes sin ingresos económicos (70\% de la muestra percibe menos que el salario mínimo interprofesional).

Por último, si bien la mayoría de los pacientes tiene una vivienda adecuada (55.4\%) un porcentaje importante (22\%) vive en la calle o en condiciones de ruina o barraquismo.

Situación clínica: La escala dispone de dos ítems que valoran la frecuencia, duración y grado de discapacidad de la enfermedad en el paciente. Se trata de una adaptación de la escala FIM (o MIF en portugués), escala de independencia 
funcional, que estima los niveles de discapacidad cualquiera que sea su origen o naturaleza.

$\mathrm{Al}$ analizar los datos relativos a la salud de la muestra, hay un predominio de la enfermedad crónica $(55,4 \%)$ pero que no afecta a la funcionalidad, ya que los pacientes psiquiátricos tienen un porcentaje de funcionalidad sobre las actividades de la vida diaria (AVD) bastante alto. (44,6\%)

Trabajo Social Clínico: Los ítems referentes al TSS son los referentes a la intervención, al tiempo y a los recursos (y su disponibilidad).

Son aspectos importantes en las hospitalizaciones y que marcarán la planificación del alta hospitalaria, así como aportarán información sobre la complejidad de nuestra intervención.

La escala discrimina entre: la intervención, el número de asistencias, el número de contactos o coordinaciones, la disponibilidad de las respuestas y el tiempo de resolución social.

En la tabla 2 podemos observar los diferentes porcentajes referentes a la actividad de la intervención social que se clasifican en la Escala ECISACHBCN PSMAR.

Tabla 2: INTERVENCIÓN SOCIAL

\begin{tabular}{|l|c|c|}
\hline & Frecuencia & $\begin{array}{c}\text { Porcentaje } \\
\text { Valido }\end{array}$ \\
\hline Información /orientación/acompañamiento psicosocial & 15 & 18,1 \\
\hline Asesoramiento social /información & 12 & 14,5 \\
\hline $\begin{array}{l}\text { Información/orientación, acompañamiento psicosocial, } \\
\text { derivación a un equipamiento/devolución social o } \\
\text { elaboración de un informe social interno o externo }\end{array}$ & 40 & 48,2 \\
\hline $\begin{array}{l}\text { Información/orientación, acompañamiento psicosocial, } \\
\text { derivación hasta dos equipamientos/devolución social } \\
\text { o elaboración de un informe social interno o externo } \\
\text { y/o realización de una gestión fuera de la institución } \\
\text { (visita domiciliaria / acompañamiento a gestiones } \\
\text { administrativas/reuniones externas) }\end{array}$ & 12 & 14,5 \\
\hline $\begin{array}{l}\text { Información/orientación, acompañamiento psicosocial, } \\
\text { derivación a más de dos equipamientos/devolución social } \\
\text { o elaboración de un informe social interno o externo } \\
\text { y/o realización de una gestión fuera de la institución } \\
\text { (visita domiciliaria / acompañamiento a gestiones } \\
\text { administrativas/reuniones externas }\end{array}$ & 4 & 4,8 \\
\hline \begin{tabular}{l} 
Total \\
\hline
\end{tabular} & 83 & 100,0 \\
\hline
\end{tabular}


En cuanto al número de asistencias, las atenciones se sitúan en la franja inicial de la tabla, con dos atenciones en un $41 \%$ de los casos, posiblemente explicable por ser un centro de agudos, de ingresos breves, donde las atenciones sociales disminuyen, potenciándose las de otros profesionales. De la misma manera, el número de contactos con la comunidad se sitúa en la misma franja, situándose entre los dos y cuatro contactos o coordinaciones (42.1\%).

En cambio, el ítem se mide la disponibilidad de las respuestas institucionales, relevante para la planificación del alta hospitalaria, que hay dificultades graves en estas, no habiendo el recurso necesario, adecuado para el paciente psiquiátrico en el ámbito de lo social (sobre todo en el ámbito residencial).

Esta falta de respuesta de la comunidad para cubrir las necesidades sociales hace que el tiempo de resolución social se alargue, situándose en la franja de más de 15 días la resolución del caso, alargándose también la estancia hospitalaria.

Como en los dos anteriores ítems hay una cierta polaridad con el ítem menos complejo, seguramente correspondiente a los pacientes con buen soporte o familia ( $25,3 \%$ frente al $32,5 \%$ ).

Tras la descripción de los ítems de la escala, confrontaremos las hipótesis planteadas al inicio del estudio con los datos estadísticos del estudio, mediante la asociación de las diferentes variables implicadas.

\section{Comparación entre estancia hospitalaria, complejidad social y apoyo familiar}

Tras la descripción de los ítems de la escala, confrontaremos las hipótesis planteadas al inicio del estudio con los datos estadísticos del estudio, mediante la asociación de las diferentes variables implicadas.

\section{Hipótesis I}

Existe una relación entre la complejidad de la intervención y la duración de la estancia hospitalaria

Para demostrar esta hipótesis, en primer lugar, hemos realizado un análisis univariante entre la duración del ingreso con las posibles variables asociadas, que, según la literatura consultada (Seva-Diaz y Seva-Fernández, 2003) en el ámbito de la psiquiatría son: la edad, el diagnóstico y la complejidad social, utilizando las técnicas estadísticas adecuadas (ANOVA y correlaciones).

Tras ese análisis se utilizaron técnicas de regresión para determinar qué factores afectan independientemente la variable de estudio (duración del ingreso) 
Para ello hemos analizado en primer lugar dos variables cuantitativas mediante una correlación de Pearson, la duración (días) y el total de la Escala ECISACH, y a continuación dos variables más, la edad y la duración de los días.

Tabla 3: CORRELACIÓN DE PEARSON DE LAS VARIABLES EDAD, DURACIÓN DEL INGRESO Y DEL TOTAL DE LA ESCALA ECISACH-BCN PSMAR

\begin{tabular}{|c|c|c|c|}
\hline & & DÍAS & EDAD \\
\hline \multirow{4}{*}{ DÍAS } & Correlación Pearson & 1 &,$- 235^{*}$ \\
\hline & Sig. (bilateral) & & ,032 \\
\hline & $\mathrm{N}$ & 83 & 83 \\
\hline & Correlación Pearson &,$- 235^{*}$ & 1 \\
\hline \multirow[t]{3}{*}{ EDAD } & Sig. (bilateral)* & ,032 & \\
\hline & $\mathrm{N}$ & 83 & 83 \\
\hline & & dias & TOTAL \\
\hline \multirow{4}{*}{ DÍAS } & Correlación Pearson & 1 & , 196 \\
\hline & Sig. (bilateral) & & ,076 \\
\hline & $\mathrm{N}$ & 83 & 83 \\
\hline & Correlación Pearson & ,196 & 1 \\
\hline \multirow[t]{2}{*}{ TOTAL } & Sig. (bilateral)* & ,076 & \\
\hline & $\mathrm{N}$ & 83 & 83 \\
\hline
\end{tabular}

* La correlación es significativa al nivel 0,05 (bilateral)

El resultado en el caso de correlación días de ingreso-complejidad, si bien no ha sido significativo estadísticamente (0'076) si sugiere una tendencia, y dado nuestra hipótesis a priori hemos decidido realizar un multivariante para descartar factores de confusión.

A continuación, realizamos una ANOVA univariante para conocer si hay relación entre los días de ingreso (variable dependiente cuantitativa) y los diagnósticos (variable independiente categórica). El valor de F es alto, por lo que significa que estas dos variables están relacionadas (tabla 4). 
Tabla 4: ANOVA

\begin{tabular}{|l|c|c|c|c|c|}
\hline \multicolumn{7}{|c|}{ ANOVA } \\
\hline & $\begin{array}{c}\text { Sumade } \\
\text { cuadrados }\end{array}$ & $g l$ & $\begin{array}{c}\text { Media } \\
\text { cuadrática }\end{array}$ & F & Sig. \\
\hline Inter-grupos & 2141,626 & 3 & 713,875 & 4,205 &, 008 \\
\hline Intra-grupos & 13412,398 & 79 & 169,777 & & \\
\hline Total & 15554,024 & 82 & & & \\
\hline
\end{tabular}

Tras este primer análisis, y viendo la posible influencia de la complejidad de la intervención en la estancia, realizamos una regresión múltiple (variables independientes diagnóstico, edad y complejidad y variable dependiente días de ingreso)

Tabla 5: MODELO DE REGRESIÓN

\begin{tabular}{|c|c|c|c|c|c|c|}
\hline \multirow{2}{*}{ Modelo } & \multicolumn{2}{|c|}{$\begin{array}{l}\text { Coeficiente no } \\
\text { estandarizado }\end{array}$} & \multirow{2}{*}{$\mathrm{t}$} & \multirow{2}{*}{ Sig. } & \multicolumn{2}{|c|}{$\begin{array}{c}\text { Intervalo de } \\
\text { Confianza para B } \\
\text { al } 95 \%\end{array}$} \\
\hline & B & $\begin{array}{l}\text { Error } \\
\text { típ. }\end{array}$ & & & $\begin{array}{l}\text { Límite } \\
\text { inferior }\end{array}$ & $\begin{array}{l}\text { Límite } \\
\text { superior }\end{array}$ \\
\hline (Constante) & $\begin{array}{c}-4,007 \mathrm{E}- \\
013 \\
\end{array}$ & 12,739 &, 000 & 1,000 & $-25,366$ & 25,366 \\
\hline EDAD &,- 139 & ,082 & $-1,703$ & ,093 &,- 301 & ,023 \\
\hline $\begin{array}{l}\text { COMPLEJIDAD DE LA } \\
\text { INTERVENCION } \\
\text { SOCIAL }\end{array}$ & ,305 & , 154 & 1,985 & ,051 &,- 001 & ,611 \\
\hline $\begin{array}{l}\text { DIAGNOSTICO } \\
\text { REDUCIDO21 }\end{array}$ & 22,824 & 14,072 & 1,622 & , 109 & $-5,197$ & 50,845 \\
\hline $\begin{array}{l}\text { DIAGNOSTICO } \\
\text { REDUCIDO31 }\end{array}$ & 8,043 & 16,844 & ,478 & ,634 & $-25,497$ & 41,584 \\
\hline $\begin{array}{l}\text { DIAGNOSTICO } \\
\text { REDUCIDO41 }\end{array}$ & 11,369 & 15,133 & ,751 & ,455 & $-18,764$ & 41,503 \\
\hline $\begin{array}{l}\text { DIAGNOSTICO } \\
\text { REDUCIDO51 }\end{array}$ & 7,290 & 18,668 & ,390 & ,697 & $-29,883$ & 44,462 \\
\hline
\end{tabular}


Los análisis regresivos consisten en estudiar la relación entre variables, para conocer si existe una asociación, cuanta fuerza tiene (coeficiente de correlación) y su forma y /o modelo.

En este caso vemos que la complejidad de la intervención es la variable que es estadísticamente significativa en relación con la estancia y su duración, por lo que podemos decir que la primera hipótesis se verifica: hay una relación entre la complejidad de la intervención y la estancia hospitalaria.

\section{Hipótesis II}

La familia es un factor protector frente a la complejidad de la intervención social: los pacientes con familia presentan menor complejidad de la intervención social en los ingresos hospitalarios y al revés, los pacientes sin familia presentan mayor complejidad Se realiza un análisis univariante (tstudent) entre la presencia de familia y la complejidad.

$\mathrm{Al}$ analizar las dos variables vemos que la media de los pacientes sin familia es superior a la media de los pacientes con familia de forma estadísticamente significativa, $(\mathrm{m}=39.30 \mathrm{sd} .8 .019 \mathrm{vs} \mathrm{m}=31.87 \mathrm{sd} .9 .131 ; \mathrm{t}=3.4430, \mathrm{p}<0.001)$, por lo que podemos decir que la hipótesis dos también se verifica.

Tabla 6: ANÁLISIS COMPLEJIDAD Y PRESENCIA DE FAMILIA

\begin{tabular}{|c|c|c|c|c|c|c|}
\hline & & $\mathrm{N}$ & MEDIA & $\begin{array}{l}\text { Desviación } \\
\text { Estándar }\end{array}$ & \multicolumn{2}{|c|}{ Std. Media Error } \\
\hline & $\begin{array}{l}\text { Pacientes sin } \\
\text { familia }\end{array}$ & 23 & 39,30 & 8,019 & \multicolumn{2}{|c|}{1,672} \\
\hline TOTAL & $\begin{array}{l}\text { Pacientes con } \\
\text { familia }\end{array}$ & 60 & 31,87 & 9,131 & \multicolumn{2}{|c|}{1,179} \\
\hline & & \multicolumn{2}{|c|}{$\begin{array}{l}\text { Test de Levene para } \\
\text { igualdad de varianzas }\end{array}$} & & & \\
\hline & & $\mathrm{F}$ & Sig. & $\mathrm{t}$ & df & $\begin{array}{c}\text { Sig. } \\
\text { (2-tailed) }\end{array}$ \\
\hline & $\begin{array}{l}\text { Igualdad de } \\
\text { varianzas } \\
\text { asumidas }\end{array}$ & 1,261 & ,265 & 3,430 & 81 & ,001 \\
\hline TOTAL & $\begin{array}{l}\text { Igualdad de } \\
\text { varianzas no } \\
\text { asumidas }\end{array}$ & & & 3,635 & 45,141 &, 001 \\
\hline
\end{tabular}




\section{Discusión}

Los resultados obtenidos en este estudio coinciden con los estudios realizados en diferentes campos de la salud, donde se evidencia que las relaciones sociales influyen en los resultados de la salud de los adultos (Holt-Lunstad, Smith y Layton, 2010): la estancia hospitalaria tiene relación con la complejidad de la intervención social y la presencia de apoyo familiar es un factor protector frente a la complejidad de la intervención social.

El apoyo social aumenta la capacidad de los pacientes para hacer frente y adaptarse a la vida diaria (Gutiérrez-Maldonado, Caqueo-Urízar, Ferrer-García y Fernández-Dávila, 2012) y si lo traspasamos al campo de la salud mental, nos encontramos que los pacientes con apoyo socio-familiar tienen mejores condiciones de vida y presentan menores ingresos hospitalarios que aquellos que carecen de este apoyo (Norman, Malla y Manchanda, 2005).

El apoyo familiar también influye en el curso de las enfermedades crónicas (como las enfermedades mentales graves), y puede convertirse en un factor de protección o de riesgo según se cuente con él o no (Ponce, López, Velázquez, Márquez, Bellido y Cruz, 2007). Por lo tanto, los diferentes estudios ya nos presentaban la influencia de estos determinantes sociales en la salud (mental) de los pacientes.

La aportación de este estudio al tema que tratamos es cómo estos determinantes influyen o no en la complejidad de la intervención del Trabajador/a Social Sanitario/a, el peso que tiene el apoyo familiar y social en el nivel de complejidad de la intervención, y si esto a su vez influye en la estancia hospitalaria. Los resultados nos indican que los pacientes con menor apoyo familiar y menor disponibilidad de relaciones sociales afectivas tienen un nivel de complejidad más alto que el resto de los pacientes.

Así mismo los análisis estadísticos nos indican que la complejidad de la intervención social y la duración del ingreso hospitalario están relacionadas, por lo que podemos decir que el apoyo familiar y social de los pacientes determinara el nivel de intervención social (complejidad) y la duración del ingreso. Esto es relevante ya que conocer estos determinantes nos permitiría desarrollar estrategias adecuadas, protocolos de intervención, sobre el colectivo que presenta una mayor complejidad en la intervención, la persona sin apoyo familiar

Ello podría significar una reducción en la estancia hospitalaria, pudiendo ser objeto de siguientes estudios. Estudiar entonces el apoyo social y familiar de los pacientes es un factor importante para predecir recursos necesarios para el tratamiento de los pacientes en el ámbito hospitalario, siendo un factor prioritario en las valoraciones psicosociales de los pacientes. La escala ECISACH-BCN PSMAR es un buen instrumento para medir y evaluar la complejidad de la 
intervención social, su utilización en el estudio ha posibilitado realizar un análisis descriptivo de los factores sociales, clínicos y característicos de la intervención social, por lo que recomendamos su incorporación a la práctica cuotidiana del profesional del Trabajo Social Sanitario.

\section{Limitaciones}

Este estudio tiene limitaciones de carácter temporal y estructural. En cuanto a la limitación temporal, el estudio se ha realizado durante 4 meses y pudiera ser que la muestra recogida no sea significativa.

La limitación estructural tiene que ver con la muestra también, ya que ésta tiene el sesgo de que ya son pacientes derivados al TSS, por lo que alguien (profesionales, la familia, o el propio usuario) ya han detectado un riesgo social.

Para poder realizar el estudio sin estigmas, el número total de personas atendidas por el TSS debería ser el número total de ingresos en la unidad, todas las personas que ingresan en la unidad deberían ser valoradas por el TSS y así poder analizar mediante la escala ECISACH-BCN PSMAR las características de nuestra intervención.

\section{Bibliografía}

BARKER, R. (1991). The Social Work Dictionary. Silver Spring, MD: National Association of Social Workers.

Caballero, G.E., Moreno, G.M., Sosa, C. M.E., Mitchel, F.E., Vega, H.M., ColumbiÉ, P. (2012). Los determinantes sociales de la salud y sus diferentes modelos explicativos. INFODIR, 8 (15).

Colom, D. (2012). El Trabajo social sanitario como herramienta de gestión. Sedisa, $14-24$.

Colom, D. (2012). El diagnóstico social sanitario. Aval de la intervención y seña de identidad del trabajo social sanitario. Barcelona: UOC.

Cordero, N. y Blanco, J. (2004). ¿Trabajo social sin fundamento? Aportaciones del paradigma de la complejidad a la epistemología del Trabajo Social. Portularia, 4, 407-412.

DAHLGReN, G. y WhiteHEAD, M. (1992). Policies and strategies to promote equity in health. Copenhagen: WHO Regional Office for Europe. EUR/ICP/RPD 414 (2).

EsCARTín, M.J. (1992). El sistema familiar y el trabajo social Alternativas: Cuadernos de trabajo social, 1, 55-75.

Galán, A., Blanco, A. Pérez, M.A. (2000). Análisis del concepto de Conducta de Enfermedad: Un acercamiento a los aspectos psicosociales del enfermar. Anales de psicología, 16, (2), 157-166. 
Garcés Trullendue, E.M. (2010). El Trabajo Social en Salud Mental. Cuadernos de Trabajo Social, 23, 333-352.

Gutiérrez, J., Caqueo, A., Ferrer, M., \& Fernández, P. (2012). Influencia de la percepción de apoyo y del funcionamiento social en la calidad de vida de pacientes con esquizofrenia y sus cuidadores. Psicothema, 24(2), 255-262.

HERNÁNDEZ R.; FERNÁNDEZ, C. y BAPTISTA, P. (2007). Metodología de la Investigación. Ciudad de México: McGraw-Hill. 6 edición.

Holt-Lunstad, J., Smith, T.B. y Layton, J.B. (2010). Social Relationships and Mortality Risk: A Meta-Analytic Review. PLoS Medicine 7 (7) https://doi. org/10.1371/journal.pmed.1000316

Ituarte Tellaeche, A. (coord.) (2017). Prácticas del trabajo social clínico. Valencia: Nau Llibres.

LALONDE, M. (1974). A new perspective on the health of Canadians. Ottawa: Minister of Supply and Services Canada. Retrieved from Public Health Agency of Canada website: http://www.phac-aspc.gc.ca/ph-sp/pdf/perspect-eng.pdf

LAmatA, F. (1994). Una perspectiva de la política sanitaria 20 años después del informe Lalonde. Gaceta Sanitaria, 8, 189-94. https://doi.org/10.1016/ S0213-9111(94)71192-4

MEDRANO,J. (2014). Boticarium. Revista de la Asociación Española de Neuropsiquiatría, 34 (121), 175-194. https://doi.org/10.4321/S0211-57352014000100011

Ministerio DE SANidAD y CONSUMO (1998). Análisis y Desarrollo de los GDR en el Sistema Nacional de Salud. Madrid: Ministerio de Sanidad y Consumo

Morín, E. (2004). Introducción al pensamiento complejo. México: Gedisa

Morro, L., González, S., Pineda, I., Cañete, M.J., Casals, A., Vallvé, M., Campos, S., Conti, M., Moreno, A., Comas, M. y Prats, A. (2017). Trabajo social sanitario y complejidad: traducción al español y validación a nuestro medio de la escala de complejidad de la intervención social con adultos en un contexto hospitalario (ECISACH), la escala ECISACH-BCN PSMAR. Revista Aghatos. Atención Socio sanitaria y Bienestar, 1, 48-54.

Norman, RMG, Malla, AK, Manchanda, R, Harricharan, R, TAKHAR, J \& NORTHCOTT, S. (2005). Social support and three-year symptom and admission outcomes for first episode psychosis. Schizophrenia Research, 80, 227-344. https://doi.org/10.1016/j.schres.2005.05.006

OMS (2007). Commission on social determinants of health. A conceptual framework for action on the social determinants of health. http://www.who.int/sdhconference/resources/ConceptualframeworkforactiononSDH_eng.pdf

OMS (2017). Plan de acción sobre la salud mental 2013-2020. http://apps.who.int/ iris/bitstream/10665/97488/1/9789243506029_spa.pdf

Peralta, V., Basterra, V., Zandio, M. y Cuesta, MJ. (2008). Psicosis cicloides: etiopatogenia, características clínicas y nosología. Aula Médica Psiquiatría, 10(1), 5-3. 
Ponce González, J. M., Velázquez Salas, A., Márquez Crespo, E., lópez Rodríguez, L., \& Bellido Moreno, M. ${ }^{a}$ L. (2009). Influencia del apoyo social en el control de las personas con diabetes. Index de Enfermería, 18(4), 224-228. https://doi.org/10.4321/S1132-12962009000400002

SERAFIn, M.R. y EsPiritu, M.I, (2013). Creación de validación de una Escala de complejidad de intervención desde el trabajo social sanitario, con adultos en un contexto hospitalario (ECISASH). Revista Aghatos. Atención Socio sanitaria y Bienestar, 1, 42-55.

Seva-Díaz, A., SeVA-Fernández, A. (2003). Los GRDs Psiquiátricos: Una investigación pendiente. European Psychiatry Journal, 17 (1), 49-63. https://doi. org/10.4321/S1579-699X2003000100006

VALLEJO, J. (2002). Introducción a la psicopatología y la psiquiatría. Barcelona: Elsevier Masson. 